\title{
An Investigation into Personality, Stress and Sleep with Reports of Hallucinations in a Normal Population
}

\author{
-Hallucinations in Normal Population
}

\author{
Jim Barnes ${ }^{1}$, Lucy Koch ${ }^{1}$, Chloe Wilford ${ }^{1}$, Laura Boubert ${ }^{2}$ \\ ${ }^{1}$ Department of Psychology, Oxford Brookes University, Oxford, United Kingdom; \\ ${ }^{2}$ Department of Psychology, University of Westminster, London, United Kingdom. \\ Email: jim.barnes@brookes.ac.uk \\ Received March 28 ${ }^{\text {th }}, 2011$; revised May $6^{\text {th }}, 2011$; accepted June $13^{\text {th }}, 2011$.
}

\begin{abstract}
Emotion, especially anxiety, has been implicated in triggering hallucinations. Sleep behaviour has also been reported to have a modest influence on the judgments that lead to hallucinatory experiences. We report an investigation on the prediction of hallucinatory predisposition which explored emotion and associated processes (stress, personality and sleep behaviour) using a questionnaire survey in a student population $(\mathrm{N}=127)$. Findings indicated significant associations between perceived stress levels and sleep, with stress and being a significant predictor of the hallucinatory experience. In addition there was a predictive relationship between the proneness to hallucinate and schizotypal personality traits, characterised by the subscale of cognitive disorganisation and unusual experiences. Stress and anxiety together with personality may need to be considered in the understanding of hallucinatory experience.
\end{abstract}

Keywords: Hallucinations, Normal Population, Personality

\section{Introduction}

Hallucinations are perceptions in the absence of an external stimulus (Bentall, 1990). The phenomena can occur in any sensory modality and can arise through neurologic disease, psychopathology, and the use of recreational drugs. Indeed hallucinations are widely accepted to be symptomatic of a psychotic disorder and like schizophrenia they occur within a clinical setting, however recently they have been investigated in healthy people who have a predisposition for visual hallucinations (Johns, 2005; Johns \& Os van, 2001). Though not psychotic, these people can still have hallucinations as seen in clinical populations. Along these lines, studies evaluating the prevalence of hallucinations in the general public continually observe, on average, that $35 \%-40 \%$ of individuals admit that they have had some experience of visual hallucinatory phenomenon (Barrett \& Etheridge, 1992; Ohayon, 2000; Posey \& Losch, 1983; Tien, 1991). Higher degrees of negative affect, particularly depression, anxiety, and stress, have been repeatedly and consistently reported to correlate with hallucinations (Delespaul, Vries de, \& Os van, 2002; Freeman \& Garety, 2003). Observations like these suggest that emotional distress might have a direct influence on the onset of hallucinations or that some of the mechanisms that cause hallucinations may be managed or moderated by affective arousal (Slade \& Bentall, 1988).

Studies have also focused on the relationship of personality traits and hallucinatory experiences using non-clinical samples. The Launay-Slade Hallucination Scale (Launay \& Slade, 1981), a scale that is used to measure tendency to visually hallucinate, correlates with scores on neuroticism and openness to experience features on the NEO Personality Inventory (Costa \&
McCrae, 1992), which suggests that certain personality traits have an impact on the occurrence of hallucinations (Laroi \& Van der Linden, 2005). In fact, a certain personality trait that reportedly influences the occurrence of hallucinations in healthy people is schizotypy (Claridge, Clark, \& Davis, 1997). Schizotypy is explained as being a psychological idea that demonstrates a constant sequence of personality traits and encounters that are connected with psychosis and, specifically, schizophrenia (Claridge, 1997). Schizotypy is different from schizophrenia, as increased levels of schizotypy do not mean that a person is in poor health (Claridge \& Beech, 1995), and certain traits are considered to be advantageous as they pertain to creativity and academic accomplishment (Nettle, 2006).

Not unlike hallucinations, schizotypy is seen as a part of the personality that has a normal distribution in the general population (Claridge \& Beech, 1995). The personality characteristics that define schizotypy can be measured using the Oxford-Liverpool Inventory of Feelings and Experiences (Mason, Claridge, \& Jackson, 1995) and are often broken up into four features: unusual experiences, cognitive disorganisation, introverted anhedonia and impulsive non-conformity (Bentall, Claridge, \& Slade, 1989; Claridge et al., 1996). Unusual experiences refers to perceptual, hallucinatory and magical thinking; cognitive disorganisation refers to problems with attention, concentration and decision making; introvertive anhedonia refers to a lack of enjoyment from social activities as well as a dislike of physical and emotional intimacy and impulsive non-conformity refers to violent, reckless and self-abusive behaviours.

Individuals scoring highly on measures of schizotypy are also prone to "micro-sleeps". "Micro-sleeps" are the result of hyper-arousal coupled with extreme stress and involve intrusions of stage one sleep into waking consciousness (Oswald, 
1962). Stage one sleep is the transition between wakefulness and sleep and can be accompanied with sensory experiences such as vivid hallucinations (Ohayon, Priest, Caulet, \& Guilleminault, 1996). This suggests that individuals with high levels of schizotypy personality characteristics may have a predisposition to hallucinate in times of extreme stress. Stressful events have been shown to activate the predisposition to hallucinate in vulnerable individuals (Nuechterlein \& Dawson, 1984) and individuals predisposed to hallucinate report higher levels of depression, anxiety and stress, (Paulik, Bafcock, \& Maybery, 2006). Also, there have been several studies that have concluded that poor sleep quality is frequently connected to a chaotic and high stress lifestyle and, most especially, has an impact on women living in western cultures (Rajaratnam \& Arendt, 2001; Soares, 2005). The results of polysomnography studies support this finding and suggest that generalized anxiety and worry in otherwise healthy people can cause significant sleep problems (Fuller, Waters, Binks, \& Anderson, 1997). Along the same line, adolescents who are chronic poor sleepers show more noticeable behaviours and feelings of stress than good sleepers (Kirmil-Gray, Eagleston, Gibson, \& Thoresen, 1984). This is most notable in a student population who are susceptible to a great number of stressors (Medeiros, Mendes, Lima, \& Araujo, 2001).

In conclusion, research on the occurrence of hallucinatory experiences has established that they are not only occurring in the general population but also have many associative factors which contribute to a person's proneness to hallucinate. This study investigated the link between these associations particularly the possible relationship between the occurrence of hallucinatory experiences and schizotypy personality characteristics, sleep quality and stress in a student population. It is hypothesised that stress and sleep quality will predict hallucinatory predisposition and that high levels of schizotypy may increase the likelihood of the hallucinatory experience.

\section{Methods}

\section{Participants}

The study was passed by the local university ethics committee. Participant recruitment was done via email to university students at Oxford Brookes University, Oxford, informing them of the study. Participants recruited were 117 students (75 female, 42 males) who were tested over a period of five weeks. Their mean age was 22.3 years $(\mathrm{SD}=5.3)$

\section{Measures}

Participants provided basic demographic information (age, gender) and completed the following questionnaires. All questionnaires are well known in the literature and have strong reliability and validity for the both hallucinatory and sleep research (Barnes et al., 2010).

\section{The Undergraduate Stress Questionnaire (Crandall, Preisler, \& Aussprung, 1992)}

Life stress was measured with the Undergraduate Stress Questionnaire (USQ) (Crandall, Preisler, \& Aussprung, 1992). The USQ is an 83 -item checklist designed to measure stress among university students based on life-events life events they have experienced in the semester. Each check mark is tallied for a total score on the USQ. The checklist includes events such as "death of a family member" and "lack of money". The USQ has been found to correlate positively with physical symptoms and negatively with mood (Crandall et al., 1992)

\section{Revised Hallucination Scale (RHS)}

This is a 24-item questionnaire based upon the revised Launay-Slade Hallucination Scale (Launay \& Slade, 1981; Morrison, Wells, \& Nothard, 2000). It incorporates additional items measuring predisposition to visual hallucination, predisposition to auditory hallucinations, vividness of imagery and daydreaming in order to examine distinctions between these phenomena in greater detail. It retained the revised method of scoring, allowing items to be endorsed using a 4-point scale to measure frequency $(1=$ never, $2=$ sometimes, $3=$ often, $4=$ almost always) rather than a forced true/false response.

\section{The Pittsburgh Sleep Quality Index (PSQI) (Buysse et al. 1989)}

Sleep quality during the previous month and to discriminate between good and poor sleepers the (PSQI) was administered. Sleep quality is a complex phenomenon that involves several dimensions, each of which is covered by the PSQI. The covered domains include Subjective Sleep Quality, Sleep Latency, Sleep Duration, Habitual Sleep Efficiency, Sleep Disturbances, Use of Sleep Medications, and Daytime Dysfunction (Buysse, Reynolds, Monk, Berman, \& Kupfer, 1989).

\section{The Oxford-Liverpool Inventory of Feelings and Experiences (O-LIFE; Mason et al., 1995)}

Previous research has shown that O-LIFE has high internal consistency (Mason et al., 1995) and high test-retest reliability (Burch, Steel, \& Hemsley, 1998). So in order to maintain validity and reliability, the whole O-LIFE questionnaire was used. The questionnaire assesses the following four dimensions: Unusual Experiences: reflects the positive symptoms of psychosis, and consists of items assessing magical thinking, unusual perceptual aberrations, and hallucinatory experiences (e.g., "When in the dark do you often see shapes and forms even though there is nothing there?"; "Are your thoughts sometimes so strong that you can almost hear them?"). Cognitive Disorganization: reflects the disorganized aspect of psychosis, and consists of items assessing difficulties with concentration and decision making, as well as social anxiety (e.g., "No matter how hard you try to concentrate do unrelated thoughts always creep into your mind?"; "Are you sometimes so nervous that you are blocked?"). Introvertive Anhedonia: reflects the negative aspects of psychosis, and consists of items assessing the lack of enjoyment from social contact, physical activities, coupled with aversion to emotional and physical intimacy (e.g., "Are you much too independent to get involved with other people?"; "Are people usually better off if they stay aloof from emotional involvements with people?"). Impulsive Non-conformity: consists of items assessing aggressive, anti-social and impulsive behaviour (e.g., "Were you ever greedy by helping yourself to more than your share of anything?"; "Do you ever feel the urge to break or smash things?"). The score for each scale is given by the accumulated points across the items within the scale and higher scores correspond to higher schizotypy. 


\section{Results}

Mean questionnaire scores are shown in Table 1. Using SPSS, score distribution histograms showed normal distribution and Pearson's correlations and standard multiple regressions were used. Correlation scores are shown in Table 2. There was no evidence that Age was significantly correlated with any measure. With respect to the schizotypy scale, the correlations revealed a significant correlation between the scores on the hallucination scale and Unusual Experiences, Cognitive Disorganisation and Impulse Non Conformity. However there is no significant correlation between scores on the hallucination scale and Introverted Anhedonia. Stress score and sleep scores were also positive correlate with the hallucinatory experience.

A linear regression analysis to test the extent to which stress, sleep, age and schizotypy could predict differences in the hallucination score (see Table 3). In these analyse, no significant contribution was found for age or sleep, as a result these variables were not included in subsequent regression analyses. The regression analysis results showed that, stress scores, Unusual Experiences and Cognitive Disorganisation was significantly positively correlated and were predictive of hallucinatory experiences.

\section{Discussion}

In partial accordance with our main hypotheses, aspects of schizotypy, and stress, were predictive of hallucinatory temperament. High levels of stress, unusual experiences, and cognitive disorganisation were correlated with hallucinatory experiences. Stress was the most prominently related which was consistent with earlier reports of clinically-based research underscoring the impact of emotional processes on the occurrence of hallucinations (Fowler, Garety, \& Kuipers, 1995; Slade, 1973). There was also a clearly established correlation between the quality of sleep and tendency to experience hallucinations. Hallucinatory occurrences related to sleep were previously found in normal populations (Laroi, Van der Linden, \& Marczewski, 2004) and in clinical observations in which symptoms related to poor sleep and levels of arousal were associated with hallucinatory incidents (Barnes, Connelly, Wiggs,
Boubert, \& Maravic, 2010). Not only does the ability to sleep well contribute to physical and emotional health (Pilcher, Ginter, \& Sadowsky, 1997), it also serves as a predictor of anxiety for the individuals in the study. Participants reported continuing emotional disturbances, which in debriefing appeared to be caused by their concerns that they might hallucinate while trying to fall asleep. It is conceivable, then, that individuals experience hallucination-like episodes might begin to have difficulties sleeping due to an increase in anxiety that they might hallucinate when going to sleep. This contribution that anxiety has to the hallucinatory experience must be more deeply examined, since it has been revealed that degrees of anxiety tend to be lower as a hallucination ends, not higher, and just before hallucination starts, we see a rise in anxiety, suggesting there may be some contribution in the formation of the hallucination (Delespaul et al., 2002).

The occurrence of hallucinations and schizotypy personality traits had a normal distribution within the population, confirming the notion that hallucinations are widely experienced in general population (Claridge \& Beech, 1995). Introverted anhedonia did not appear to predict hallucinations. This indicates that self-reported hallucination tendencies do not rely on perceived sociability and that experiencing hallucinations has little association with finding pleasure in social and physical interaction. A positive association was also found between individuals who are prone to hallucinate and individuals who experience Cognitive Disorganisation, Unusual Experiences, and Impul sive Non-conformity subdivisions of the O-Life. This suggests that personality can be a strong predictor of the hallucination

Table 1.

Showing the mean values and standard deviation values for each of the questionnaire scores.

\begin{tabular}{ccc}
\hline Questionnaire & Mean & Standard Deviation \\
\hline Undergraduate Stress Questionnaire & 26.3 & 11.4 \\
Pittsburgh Sleep Quality Index & 6.35 & 3.60 \\
Launay Slade Hallucination Scale & 13.6 & 8.67 \\
Olife Total Score & 32.6 & 11.9 \\
\hline
\end{tabular}

Table 2.

Showing correlations of main measures.

\begin{tabular}{|c|c|c|c|c|c|c|c|c|c|}
\hline & Mean & SD & 1 & 2 & 3 & 4 & 5 & 6 & 7 \\
\hline 1. Hallucination Score & 13.69 & 8.675 & 1.000 & $.557 * *$ & $.365^{* *}$ & $.410^{* *}$ & .002 & $.251 * *$ & $.407 * *$ \\
\hline 2. Stress Score & 26.31 & 11.412 & & 1.000 & $.461 * *$ & $.317 * *$ & .038 & $.200^{*}$ & $.366^{* *}$ \\
\hline 3. Sleep Score & 6.35 & 3.602 & & & 1.000 & $.365^{* *}$ & $.305^{* *}$ & .130 & $.239 * *$ \\
\hline 4. Cognitive Disorganisation & 12.47 & 5.712 & & & & 1.000 & $.178^{*}$ & $.309 * *$ & $.381 * *$ \\
\hline 5. Introvertive Anhedonia & 4.81 & 4.423 & & & & & 1.000 & -.142 & .005 \\
\hline 6. Impulsive Non-Conformity & 9.20 & 3.619 & & & & & & 1.000 & $.204 *$ \\
\hline 7. Unusual Experiences & 6.13 & 5.370 & & & & & & & 1.000 \\
\hline
\end{tabular}

**Correlation is significant at the 0.01 level; *Correlation is significant at the 0.05 level $\mathrm{N}=127$. 
Table 3.

Linear regression analysis for hallucinatory experiences as measures by Launay Slade Hallucination Scale.

\begin{tabular}{ccc}
\hline & Beta & $\mathrm{t}$ \\
\hline Stress Score & .422 & $5.433^{* *}$ \\
Unusual Experiences & .163 & $2.054^{*}$ \\
Impulsive Non Conformity & .065 & .855 \\
Introvertive Anhedonia & -.041 & -.563 \\
Cognitive Disorganisation & .201 & $2.439^{*}$ \\
\hline
\end{tabular}

$\mathrm{F}(5,121)=16.14 ; \mathrm{R}$-squared $=0.406 ;$ Adjusted R-squared $=0.376 ; * *$ Significant at the 0.01 level; *Significant at the 0.05 level.

phenomenon and supports previous work that found a similar relationship (Laroi \& Van der Linden, 2005). The impulsive non-conformity was found to correlate with the hallucination scores but not to be predictive for hallucinations in the regression calculations. This finding may be explained with reference to the shared variance between the O-LIFE dimensions, so that when the effects of the other three dimensions had been taken into account, impulsive non conformity was not predictive. Thus people who experience hallucinations would be no more likely than their peers to have unstable mood, or display disorganized and socially inappropriate behaviour.

Overall, this study was able to demonstrate that stress and schizotypy were able to account for variance in hallucination scores. But we must observe that, even though we observed consistent relationships, when we consider predictor variables, there were other factors that appeared to be connected to hallucinations when isolated and examined. This means it is possible that, even though schizotypy and stress levels forecast hallucinations, there are obviously other individual differences, like motivation and awareness for instance, that must be taken into account. There were also a number of limitations which need be acknowledged before an examination of the implications of this study. The size of the sample was relatively small, and the allocation of gender was not equal. Also, the most prominent correlations might be partly the result of overlapping methods, simply because self-reported measures forecast self-report measures. The discovery that the hallucinations were not predicted by the Introvertive Anhedonia scale may be a reflection of the negative facets of psychosis and consists of items that evaluate anhedonia when socialising and suggests that withdrawal from the social environment and being depressed are not major factors in experiencing hallucinations.

In conclusion the findings of this study suggest that the levels of stress and anxiety experienced by the participants were the most influential predictors of a tendency to hallucinate. Consequently, future investigations in this area might consider rigorously examining the emotional predictors of a person's tendency to have hallucinations. This could involve more accurate measures of stress levels, for instance, testing the cortisol levels, as well as conducting interviews, and implementing daily stress diaries maintained by the participant. In addition to offering insight into the hallucinatory experience, it may also shed light on the extent to which a person's level of anxiety has an impact on their tendency to have hallucinations.

\section{References}

Barnes, J., Connelly, V., Wiggs, L., Boubert, L., \& Maravic, K. (2010). Sleep patterns in Parkinson's disease patients with visual hallucinations. International Journal of Neuroscience, 120, 564-569. doi: 10.3109/00207454.2010.494790

Barnes, J., \& David, A. S. (2001). Visual hallucinations in Parkinson's disease: A review and phenomenological survey. Journal of Neurology, Neurosurgery \& Psychiatry, 70, 727-733. doi:10.1136/jnnp.70.6.727

Bentall, R. P. (1990). The illusion of reality: A review and integration of psychological research on hallucinations. Psychological Bulletin, 107, 82-95. doi:10.1037/0033-2909.107.1.82

Barrett, T. R., \& Etheridge, J. B. (1992). Verbal hallucinations in normals. I: People who hear voices. Applied Cognitive Psychology, 6, 379-387. doi:10.1002/acp. 2350060503

Claridge, G., \& Beech, T. (1995). Fully and quasi-dimensional constructions of schizotypy. In A. Raine, T. Lencz and S. A. Mednick (Eds.), Schizotypal personality. Cambridge: Cambridge University Press. doi:10.1017/CBO9780511759031.010

Claridge, G., Clark, K., \& Davis, C. (1997). Nightmares, dreams, and schizotypy. British Journal of Clinical Psychology, 36, 377-386. doi:10.1111/j.2044-8260.1997.tb01245.x

Costa, P. T. Jr., \& McCrae, R. R. (1992). NEO PI-R professional manual. Odessa, FL: Psychological Assessment Resources, Inc.

Crandall, C. S., Preisler, J. J., \& Aussprung, J. (1992). Measuring life event stress in the lives of college students: The Undergraduate Stress Questionnaire (USQ). Journal of Behavioral Medicine, 15, 627-662. doi:10.1007/BF00844860

Delespaul, P., DeVries, M., \& Van Os, J. (2002). Determinants of occurrence and recovery from hallucinations in daily life. Social Psychiatry and Psychiatric Epidemiology, 37, 97-104. doi: $10.1007 / \mathrm{s} 001270200000$

Fowler, D., Garety, P. A., \& Kuipers, L. (1995). Cognitive behaviour therapy for psychosis: Theory and practice. Chichester: Wiley.

Freeman, D., \& Garety, P. A. (2003). Connecting neurosis and psychosis: The direct influence of emotion on delusions and hallucinations. Behaviour Research and Therapy, 41, 923-947. doi:10.1016/S0005-7967(02)00104-3

Fuller, K. H., Waters, W. F., Binks, P. G., \& Anderson, T. (1997). Generalized anxiety and sleep architecture: A polysomnographic investigation. Sleep, 20, 370-376.

Johns, L. C. (2005). Hallucinations in the general population. Current Psychiatry Reports, 7, 162-167. doi:10.1007/s11920-005-0049-9

Johns, L. C., \& Van Os, J. (2001). The continuity of psychotic experiences in the general population. Clinical Psychology Review, 21, 1125-1141. doi:10.1016/S0272-7358(01)00103-9

Kirmil-Gray, K., Eagleston, J. R., Gibson, E., \& Thoresen, C. E. (1984). Sleep disturbance in adolescents: Sleep quality, sleep habits, beliefs about sleep, and daytime functioning. Journal of Youth and Adolescence, 13, 375-384. doi:10.1007/BF02088636

Laroi, F., \& Van der Linden, M. (2005). Metacognitions in proneness towards hallucinations and delusions. Behaviour Research and Therapy, 43, 1425-1441. doi:10.1016/j.brat.2004.10.008

Laroi, F., Van der Linden, M., \& Marczewski, P. (2004). The effects of emotional salience, cognitive effort and meta-cognitive beliefs on a reality monitoring task in hallucination-prone subjects. British Journal of Clinical Psychology, 43, 221-233. doi: $10.1348 / 0144665031752970$

Launay, G., \& Slade, P. (1981). The measurement of hallucinatory predisposition in male and female prisoners. Personality and Individual Differences, 2, 221-234. doi:10.1016/0191-8869(81)90027-1

Medeiros, A. L. D., Mendes, D. B. F., Lima, P. C. F., \& Araujo, J. F. (2001). The relationships between sleep-wake cycle and academic performance in medical students. Biological Rhythm Research, 32, 263-270. doi:10.1076/brhm.32.2.263.1359 
Nettle, D. (2006). Schizotypy and mental health amongst poets, visual artists and mathematicans. Journal of Research in Personality, 40, 876-890. doi:10.1016/j.jrp.2005.09.004

Ohayon, M. M. (2000). Prevalence of hallucinations and their pathological associations in the general population. Psychiatry Research, 97, 153-164. doi:10.1016/S0165-1781(00)00227-4

Oswald, I. (1962). Sleeping and waking. Amsterdam: Elsevier.

Pilcher, J. J., Ginter, D. R., \& Sadowsky, B. (1997). Sleep quality versus sleep quantity: Relationships between sleep and measures of health, well-being and sleepiness in college students. Journal of Psychosomatic Research, 42, 583-596. doi:10.1037/11541-000

Posey, T. B., \& Losch, M. E. (1983). Auditory hallucinations of hearing voices in 375 normal subjects. Imagination, Cognition and Personality, 3, 99-113. doi:10.1016/S0022-3999(97)00004-4
Rajaratnam, S. M., \& Arendt, J. (2001). Health in a 24-h society. Lancet, 358, 999-1005. doi:10.1016/S0140-6736(01)06108-6

Slade, P. D. (1973). The psychological investigation and treatment of auditory hallucinations: A second case report. British Journal of Medical Psychology, 46, 293-296. doi:10.1111/j.2044-8341.1973.tb02254.x

Slade, P. D., \& Bentall, R. P. (1988). Sensory deception: A scientific analysis of hallucination. London: Croom Helm.

Soares, C. N. (2005). Insomnia in women: An overlooked epidemic? Archives of Women's Mental Health, 8, 205-213. doi:10.1007/s00737-005-0100-1

Tien, A. Y. (1991). Distributions of hallucinations in the population. Social Psychiatry and Psychiatric Epidemiology, 26, 287-292. doi:10.1007/BF00789221 